\title{
LOS FACTORES INTERNOS Y EXTERNOS EN LA POLITICA EXTERIOR MEXICANA (2012-2018): UNA EVALUACIÓN GENERAL
}

\author{
INTERNAL AND EXTERNAL FACTORS IN MEXICAN \\ FOREIGN POLICY (2012-2018): \\ A GENERAL EVALUATION
}

\begin{abstract}
LES FACTEURS DÉTERMINANTS INTERNES ET INTERNATIONAUX DE LA POLITIQUE ÉTRANGÈRE MEXICAINE (2012-2018): UNE ÉVALUATION GENERALE
\end{abstract}

\author{
RAfael VelázQuez Flores \\ Universidad Autónoma de Baja California \\ rafael.velazquez@uabc.edu.mx \\ Jessica L. de Alba Ulloa \\ Universidad Anáhuac México \\ jessica.dealba@anahuac.mx
}

Resumen: El objetivo de este artículo es evaluar la política exterior de México durante la administración de Enrique Peña Nieto (2012-2018). El argumento principal es que esta actividad estuvo profundamente condicionada por factores internos y externos en las diferentes etapas del sexenio. La primera parte plantea las metas de la administración peñista en materia de política exterior. La segunda divide el sexenio en cuatro etapas: de 2012 a 2014, cuando plantea las reformas estructurales que tuvieron repercusiones en la política exterior de México; de 2014 a 2016, cuando analiza los problemas internos; de 2016 a mitad de 2018, cuando apareció en la escena política Donald Trump, y de mediados de 2018 hasta el final de la administración. La última parte del texto evalúa el cumplimiento de los objetivos trazados al inicio de la administración.

Palabras clave: Enrique Peña Nieto; política exterior; México; reformas estructurales; corrupción; Donald Trump.

ABstRACt: The objective of this article is to evaluate the foreign policy of Mexico during the Enrique Peña Nieto administration. The main argument is that 
this activity was deeply conditioned by internal and external factors in different stages of the term. The first part identifies the main foreign policy goals of the Peña administration. The second one divides the term into four stages: from 2012 to 2014, which develops the structural reforms that impacted Mexico's foreign policy; from 2014 to 2016 that analyzes domestic issues; from 2016 to mid-2018 when Donald Trump appeared on the political scene; and from mid-2018 until the end of the administration. The last part evaluates the fulfillment of the objectives set at the beginning of the administration.

Keywords: Enrique Peña Nieto; foreign policy; structural reforms; Mexico; corruption; Donald Trump.

\section{Traducción de Gonzalo Celorio Morayta}

Résumé: Le but de cet article est d'évaluer la politique étrangère du Mexique sous le gouvernement d'Enrique Peña Nieto (2012-2018), laquelle a subi l'influence d'éléments internes et internationaux le long de cette période. La première partie du texte évoque les objectifs face à l'extérieur. La seconde esquisse quatre étapes: de 2012 à 2014, le président propose des réformes structurelles qui auront des effets sur sa politique étrangère; entre 2014 et 2016, il se tourne vers les problèmes internes du pays; par la suite, jusqu'au milieu de 2018, il doit réagir à l'apparition de Donald Trump sur la scène politique; la seconde moitié de cette année-là correspond à la fin du mandat. L'article analyse dans quelle mesure le gouvernement de Peña Nieto a atteint les objectifs proposés au début.

Mots clefs: Enrique Peña Nieto; politique étrangère; Mexique; réformes structurelles; corruption; Donald Trump.

Traducción de Bernardo Mabire

Fecha de recepción: diciembre de 2018

Fecha de aceptación: febrero de 2019 
L

A política exterior durante el sexenio de Enrique Peña Nieto tuvo variaciones de forma y fondo. En los primeros meses de la administración, el resultado de la elección presidencial, los consensos internos, las reformas estructurales y un ambiente internacional favorable permitieron una mayor presencia de México en los temas globales. Sin embargo, a partir de 2014, la situación interna se complicó debido a casos de violaciones a derechos humanos y situaciones de corrupción en el sistema político. Entonces, la política exterior tuvo menor importancia para el presidente mexicano.

En 2016, otros factores impactaron de manera directa en esta materia. La aparición de Donald Trump en el escenario político estadounidense encendió focos rojos en la diplomacia mexicana. Las críticas al país en los inicios de las campañas presidenciales obligaron al gobierno de Peña a responder de manera directa. En agosto de 2017, Peña Nieto invitó a Trump a México para tratar de lograr un impacto en su visión sobre el país. En noviembre de 2017, el republicano ganó la elección presidencial y ello tuvo también repercusiones en la política exterior de Peña Nieto. La administración hizo ajustes importantes.

El $1^{\circ}$ de julio de 2018, el candidato opositor, Andrés Manuel López Obrador, triunfó en forma arrolladora en la elección presidencial. Esto marcó los últimos seis meses de la presidencia de Peña Nieto. La capacidad de negociación de México aumentó debido al resultado electoral. Con ello, la administración peñista tuvo logros importantes, como la conclusión de la renegociación del Tratado de Libre Comercio de América del Norte (TLCAN).

Este artículo evalúa la política exterior de México durante el sexenio de Enrique Peña Nieto. El argumento principal es que esa actividad estuvo condicionada por diversos factores, tanto internos como externos. En la primera mitad del sexenio, los elementos endógenos tuvieron un mayor peso. En la segunda parte de la administración, el componente externo influyó de manera significativa. En los últimos seis meses del sexenio, el factor interno volvió a tener relevancia. 
El texto está dividido en tres grandes apartados. El primero plantea las principales metas que la administración peñista se propuso. La segunda parte divide al sexenio en cuatro etapas: la primera, que comprende de 2012 a 2014, en que los consensos internos permitieron alcanzar reformas estructurales que tuvieron un impacto en la política exterior de México. La segunda abarca de 2014 a 2016, cuando los problemas internos afectaron la diplomacia del país. La tercera etapa va de 2016 a mitad de 2018, cuando apareció en la escena política Donald Trump. La última comprende de mediados de 2018 hasta la finalización de la administración. El último apartado analiza los resultados obtenidos respecto a los objetivos de política exterior propuestos al inicio.

\section{DiAgNóSTICO Y PLANEACIÓN DE LA POLÍTICA EXTERIOR}

El sexenio del presidente Felipe Calderón Hinojosa (20062012) estuvo marcado por la violencia, un magro crecimiento económico y una desastrosa imagen del país en el exterior. México estaba en los titulares de los principales diarios en Estados Unidos, Canadá, Europa y de América Latina, la mayoría de las veces con noticias negativas. ${ }^{1}$ La población percibía un Estado con instituciones debilitadas, con altos gastos y pocos resultados. En este contexto, el candidato a la presidencia del Partido de la Revolución Institucional (PRI), Enrique

${ }^{1}$ El gasto en seguridad se multiplicó por siete durante su presidencia, pero las organizaciones criminales se incrementaron. La principal preocupación de la población era la inseguridad (más de 50 por ciento), seguido por desempleo y pobreza. El crecimiento promedio anual del producto interno bruto (PIB) durante el sexenio fue de 1.66\%. María Elena Romero y Oscar Rivera; "Los retos actuales de la política exterior mexicana: una revisión desde la geopolítica crítica”, Asian Journal of Latin American Studies (2013), vol. 26, núm. 3, pp. 1-28; José Tapia, "El gasto en seguridad. Observaciones de la ASF a la gestión y uso de recursos", México Evalúa, Centro de Análisis en Políticas Públicas, A.C. (sitio de internet), junio de 2011, https://goo.gl/z2dcsy 
Peña Nieto, representaba la figura del cambio bajo un nuevo priismo. Su filosofía regresaba a una política exterior tradicionalista, basada en los principios consagrados en la Constitución mexicana, con la soberanía como rectora de la política exterior. La plataforma presentada por su equipo fijaba el rumbo hacia una diversificación en las relaciones de México y el multilateralismo para temas de derechos humanos, crimen transnacional, producción de alimentos y liberalización comercial. ${ }^{2}$ Una vez ganada la elección, los documentos oficiales en los que se plasman las grandes líneas de trabajo del gobierno -el Plan Nacional de Desarrollo (PND) y el Programa Sectorial de la Secretaría de Relaciones Exteriores (SRE)plantearon un México con responsabilidad global:

Una fuerza positiva y propositiva en el mundo al servicio de las grandes causas de la humanidad. Un país cuya actuación global incorpore la realidad nacional y las prioridades internas, enmarcadas en las otras cuatro Metas Nacionales, para que éstas sean un agente definitorio de la política exterior. Una Nación que fortalezca su voz y su presencia en la comunidad internacional, recobrando el liderazgo en beneficio de las grandes causas globales. ${ }^{3}$

El regreso del PRI a la presidencia en 2012 generó nuevas expectativas en torno a la política exterior. Desde el inicio, la administración de Peña Nieto delineó una diferencia sustantiva al no incluir seguridad, migración y derechos humanos como temas prioritarios en la agenda, a diferencia de los gobiernos anteriores, pero también buscó darle un giro distinto a su política exterior. Los planes eran hacer de México un actor global responsable. El nuevo gobierno planeaba darle

${ }^{2}$ Emilio Lozoya Austin y Jorge Montaño Martínez, "Una visión de México para el futuro. Sección especial: propuestas para la próxima política exterior de México", Foreign Affairs Latinoamérica, vol. 12, núm. 2 (2012), pp. 43-51.

${ }^{3}$ Plan Nacional de Desarrollo (PND) 2013-2018, Diario Oficial de la Federación, 20 de mayo de 2013, http://goo.gl/kxDcCS 
un mayor peso al país en el concierto de naciones desde una posición más comprometida con los problemas globales. Parecía que el objetivo era lograr mayor congruencia entre la importancia geográfica y económica del Estado con el alcance de su política exterior. Para cumplir con esa meta, el presidente planté cuatro pilares: fortalecer la presencia internacional de México; ampliar la cooperación internacional; promover el valor de México en el mundo y velar por los intereses de México en el extranjero.

Otro gran objetivo fue mejorar la imagen del país en el exterior. La percepción general que se tenía afuera antes de 2012 era de violencia, debido al número de muertes producto del narcotráfico. Para ello, el nuevo gobierno estableció varias estrategias. Se pidió a los medios de comunicación no enfatizar las noticias sobre la violencia en México y se instruyó a todas las representaciones de México en el exterior a promover una imagen positiva del país. Esta política se convirtió en una prioridad al inicio de la administración.

Alineado a los cuatro pilares, el Plan Nacional de Desarrollo 2013-2018 planteaba los siguientes objetivos de política exterior:

1) Impulsar un diálogo político para fortalecer la presencia de México en el mundo y dotar de un marco jurídico claro y confiable a la interacción con otros Estados;

2) Promover el valor de México mediante la difusión económica, turística y cultural;

3) Reafirmar el libre comercio, la movilidad de capitales y la integración productiva, $y$

4) Velar por los intereses de los mexicanos en el extranjero y proteger los derechos de los extranjeros en el territorio nacional.

Por su parte, el Programa Sectorial de Relaciones Exteriores (2013) definió los objetivos, las estrategias y las líneas de acción que guiarían el comportamiento internacional del país en el sexenio de Peña Nieto. Las prioridades internacionales serían contribuir a la prosperidad nacional mediante la promoción de México como un destino atractivo para las 
inversiones, el turismo y como socio comercial confiable y plataforma de acceso a otros mercados; promover un desarrollo mediante la cooperación, la educación y la movilidad de las personas y el intercambio de conocimientos; fortalecer el Estado de derecho, la paz y la seguridad, profundizando el diálogo político con el exterior y velar por el bienestar de los mexicanos en el exterior.

El proyecto de política exterior lucía prometedor en el papel. Dado el margen que dejó el resultado de la elección, la nueva administración le otorgó prioridad a la política exterior al principio del sexenio. Sin embargo, a la mitad de éste la situación cambió. Por un lado, el país enfrentó escenarios complicados: casos de corrupción, asesinatos, la baja del precio internacional del petróleo, la devaluación del peso mexicano y la violencia generalizada por la lucha contra el narcotráfico. Por otro, la aparición de nuevos nacionalismos y protagonismos en el mundo y los conflictos en varias regiones complicaron el sistema internacional. La llegada de Donald Trump a la presidencia en Estados Unidos y la salida del Reino Unido de la Unión Europea marcaban una nueva tendencia aislacionista. Conflictos en varias regiones del mundo y nuevos liderazgos acentuaron la incertidumbre. Los contextos interno y externo afectaron la política exterior de Enrique Peña Nieto, especialmente en la última etapa de su administración.

\section{LOS PERIODOS DE LA POLÍTICA EXTERIOR}

En este sexenio se identifican cuatro periodos de la política exterior. El primero es la etapa que comprende el inicio de la administración, de 2012 a julio de 2014. El segundo va de agosto 2014 a julio 2015, etapa en la que hacen explosión varios conflictos internos. El tercer periodo abarca de la precampaña de Donald Trump en 2015 a la elección en México del $1^{\circ}$ de julio de 2018. La última etapa son los meses de nogobierno de Enrique Peña Nieto, de julio a diciembre. 
Primer periodo: del Mexican Moment al declive de la administración

La política exterior en pocas ocasiones ha sido relevante para las acciones de un gobierno -a menos que se confunda con la política comercial-. El primer mensaje enviado para esa área fue de continuidad, al designar a José Antonio Meade como secretario de Relaciones Exteriores. El nombramiento de Meade, quien fuera secretario de Estado en Hacienda y en Energía con el presidente Felipe Calderón (2006-1012), dejaba claro que el perfil de Relaciones Exteriores continuaría apuntando al reforzamiento y la ampliación de las relaciones comerciales. ${ }^{4}$ De manera paralela, el nuevo gobierno planteó reactivar las relaciones con las distintas regiones del mundo que habían permanecido estáticas durante el sexenio anterior. Como presidente electo, Enrique Peña organizó una robusta agenda de visitas al exterior. Una vez en el cargo, realizó 36 visitas al extranjero de diciembre de 2012 al 30 de junio de 2014, en todos los continentes. Los primeros años de gobierno estuvieron marcados por una actividad constante en materia de política exterior.

Destaca la relación con Estados Unidos (EEUU), con un fuerte componente en temas de seguridad. El gobierno desapareció la Secretaría de Seguridad Pública y trasladó sus funciones a la Secretaría de Gobernación, que se encargaría de ese tema en una ventanilla única, centralizando la actividad de las agencias de EEUU; esto funcionó de cuatro a seis meses. ${ }^{5}$ En mayo de 2013, el presidente estadounidense

${ }^{4}$ Así lo expresó el propio canciller cuando mencionaba que México debía alcanzar el crecimiento y la participación en el comercio internacional a través de la política exterior. Jorge Chabat, "La política exterior de México: de Calderón a Peña Nieto", en Adrián Bonilla y Grace Jaramillo (eds.), La CELAC en el escenario contemporáneo de América Latina y del Caribe, San José, Costa Rica, Flacso, 2014, p. 37.

${ }^{5}$ Con el tiempo, la mayoría de las agencias encontraron una solución alternativa. Para cuando Tlatlaya y Ayotzinapa llegaron, el gobierno de Peña Nieto necesitaba toda la ayuda que pudiera obtener. Craig A. Deare, 
Barack Obama visitó México. El discurso giró en torno a la intención de hacer de América del Norte la región más competitiva del mundo, además de tratar temas de educación, diálogo económico y emprendimiento. ${ }^{6}$ Los gobiernos dieron también continuidad a los programas de la Iniciativa Mérida, poniendo énfasis en la prevención del delito y el desarrollo social. ${ }^{7}$ El gobierno mexicano había hecho de la meta nacional "México en Paz" un objetivo de política exterior vinculado a Estados Unidos.

En agosto del mismo año, un juez ordenó la liberación del narcotraficante Rafael Caro Quintero, en prisión por el asesinato del agente de la DEA, Enrique Camarena, en 1985. Por otro lado, en septiembre, Edward Snowden, exempleado de la CIA y antiguo contratista del gobierno de EEUU, filtró información clasificada, parte de la cual revelaba que Enrique Peña había sido espiado por el gobierno de Estados Unidos durante su campaña a la presidencia. Ambos gobiernos protestaron por estas situaciones.

Finalmente, así como la relación de México con Estados Unidos ha estado definida en términos económicos y de seguridad, también lo ha estado en lo que respecta a lazos sociales derivados de la migración. El tema de la seguridad fronteriza

A Tale of Two Eagles. The US-Mexico Bilateral Defense Relationship Post Cold War, Nueva York, Rowman \& Littlefield, 2017, pp. 353-363.

${ }^{6}$ En dicha visita se formalizó el Foro Bilateral México-Estados Unidos sobre Educación Superior, Innovación e Investigación (Fobesii). Sergio Alcocer, "Las relaciones de México con América del Norte", en $1^{\mathrm{er}}$ Informe de Labores 2012-2013, México, SRE, https://www.gob.mx/cms/ uploads/attachment/file/103256/Primer_Informe_de_Labores_ SRE_2013.pdf (consulta del 12 de mayo de 2018). "Diálogo Económico de Alto Nivel”, México-sRe, s/f, https://goo.gl/BsKv6A

${ }^{7}$ Fernando Barrientos y Daniel Añorve, "México 2013: acuerdos, reformas y descontento". Revista de ciencia politica (Santiago), vol. 34, núm.1 (2014), pp. 221-247, doi: 10.4067/S0718-090X2014000100011; Continuaron las trasferencias de equipo, tecnología y capacitación acordadas para instrumentar y dar continuidad a acciones de cooperación establecidas. Las entregas acumuladas en este periodo tuvieron un valor cercano a los 50 mdd. 
ligado al tema migratorio fue una prioridad para el gobierno de Obama, quien proponía "capas de seguridad", extendiendo la seguridad nacional de EEUU hasta la frontera sur de México. Los muros físicos y virtuales, así como las deportaciones, aumentaron durante su mandato. ${ }^{8}$ La prometida reforma migratoria estadounidense no se concretó. El gobierno mexicano tampoco hizo los esfuerzos necesarios para cabildear el proceso al considerarlo un tema de política interna del vecino país.

Canadá, por su parte, es el tercer socio comercial de México y el segundo destino de las exportaciones mexicanas. ${ }^{9}$ Históricamente, Canadá ha actuado en el entendido de que no comparte fronteras con México, ni ha mostrado interés en profundizar la relación. De cualquier manera, los encuentros de alto nivel, sobre todo para cuestiones comerciales, continuaron con regularidad, ${ }^{10}$ aunque la relación fue distante. Un tema pendiente era el de la visa impuesta por el gobierno canadiense a los mexicanos desde 2009. Ello no entorpecía el comercio y las demás iniciativas con el sector privado. Entre los tres países, el TLCAN era la pieza principal. En esta época, ni Eeuu ni Canadá pensaban en modificar el

${ }^{8}$ En el año fiscal 2015 hubo un total de 333341 deportaciones, de las que $42 \%$ fueron clasificados como criminales. México destaca con $43.8 \%$ en esa categoría, seguida de Honduras con $42.2 \%$. Daniel Villafuerte, "La política migratoria en tiempos de Obama: implicaciones en la frontera sur de México", Ciencias Sociales y Humanidades, vol. 4, núm. 1 (2017), pp. 32-33.

${ }^{9}$ El intercambio comercial entre México y Canadá se elevó nueve veces, al pasar de \$4000 mdd en 1993 a $\$ 36.1$ mmd en 2014. Secretaría de Relaciones Exteriores (SRE), México y Canadá: una relación amplia y profunda, México, SRE, 14 de octubre de 2015, https://goo.gl/eTTRFV

${ }^{10}$ Existe el diálogo político, la alianza México-Canadá, el programa de trabajadores agrícolas, la cooperación consular y en materia migratoria, educación y el diálogo en seguridad. También hay encuentros de alto nivel en materia de seguridad, de migración y sobre temas multilaterales. Gobierno de México y Gobierno de Canadá, Alianza México-Canadá, reporte anual, 2012-2013, https://goo.gl/WPMdWs 
acuerdo. ${ }^{11}$ En febrero de 2014 celebraron la Cumbre de Líderes de América del Norte, en la que el ministro Harper descartó negociar el tema de las visas.

Con América Latina y el Caribe, el gobierno de Enrique Peña Nieto buscó una recomposición de las relaciones y un acercamiento aplicando el principio de no intervención. El primer viaje que Peña Nieto hizo al exterior como presidente electo fue hacia esta región. En septiembre de 2012 visitó Guatemala, Colombia, Brasil, Chile, Argentina y Perú. Buscaba mayor legitimidad interna, además de proponer una política de influencia en la región. ${ }^{12}$

Centroamérica también fue parte de la agenda de política exterior mexicana. En marzo de 2013, Peña Nieto participó en la I Cumbre México-Sistema de Integración Centroamericana (SICA) donde se trataron temas de seguridad, migración, comercio e inversión. La región es crítica para México, dada la cuantiosa migración de centroamericanos que cruzan por territorio mexicano para llegar a EEUU. Posterior a la crisis migratoria del verano de 2014, el gobierno mexicano anunció el Programa Frontera Sur, para contener los flujos, que se impulsó a través de la Iniciativa Mérida. ${ }^{13}$ En lo concerniente al Caribe, el presidente condonó a Cuba el $70 \%$ de una deuda de casi 500 (mdd) que tenía desde hacía

11 Dana Gabriel, "The Next Phase of North American Integration. NAFTA to be Swallowed up by the Trans-Pacific Partnership (TPP)?”, Global Research (sitio de internet), 2014, http://goo.gl/Fbah4f

${ }^{12}$ Chabat, art. cit., p. 35.

${ }^{13}$ La crisis derivó en la detención de más de 60000 niños y adolescentes centroamericanos en la frontera sur de EEUU. Hay además proyectos de cooperación en la región coordinados por la Agencia Mexicana de Cooperación Internacional para el Desarrollo (Amexcid). Clare Ribando Seelke y Kristin Finklea, "U.S.-Mexican Security Cooperation: The Mérida Initiative and Beyond", 7-5700. R41349, US, Congressional Research Service, 7 de mayo de 2015, https://goo.gl/QufDbG; Agencia Mexicana de Cooperación Internacional para el Desarrollo (Amexcid), Cooperación de México con Centroamérica y el Caribe, México, Amexcid, 12 de abril de 2018. https://goo.gl/xEkQ96 
15 años. ${ }^{14}$ Esta acción fue un gesto simbólico, pues la relación con Cuba reviste una importancia política por motivos ideológicos anacrónicos. ${ }^{15}$ También se dio continuidad a la Comunidad de Estados Latinoamericanos y Caribeños (CELAC). ${ }^{16}$

Otra de las iniciativas heredada del sexenio anterior que el gobierno de Peña Nieto apoyó fue la Alianza del Pacífico (AP), bloque comercial formado por Chile, Colombia, México y Perú. La AP tiene una lógica liberal de regulación del comercio, con un andamiaje institucional y jurídico que facilita la toma de decisiones. ${ }^{17}$

Con respecto a Venezuela, el presidente Peña Nieto acudió en marzo de 2013 al funeral del presidente Hugo Chávez. Sin embargo, en noviembre del mismo año, el reemplazo de Chávez, Nicolás Maduro, anunció que un avión mexicano había sido derribado por las Fuerzas Armadas de Venezuela por haber ingresado de manera ilegal a su espacio aéreo. ${ }^{18}$ Las relaciones se deteriorarían aun más en los años siguientes.

En general, las relaciones de México con la región fueron pragmáticas, pues no hubo alineación con gobiernos por mo-

${ }^{14}$ José Meléndez, "México-Cuba: una relación con altibajos", El Universal, 27 de enero de 2014, http://goo.gl/SJvKyt

${ }^{15} \mathrm{El}$ valor de las exportaciones mexicanas a Cuba aumentó a 372.6 mdd en 2013, cerca de $0.1 \%$ de las exportaciones totales de México en ese año. Mónica Vanessa Limón Rodríguez, "La relación entre México y Cuba", Comercio Exterior, vol. 64, núm. 2 (marzo-abril de 2014), p. 11, https://goo.gl/8Vx5f5

${ }^{16}$ Mecanismo creado en 2010. Celebró la primera cumbre de Jefes de Estado y de Gobierno (enero de 2013, Santiago de Chile) en la que participó el presidente Peña Nieto; también participó en la segunda (La Habana, enero de 2014).

${ }^{17}$ Michel Leví Coral y Giulliana Reggiardo, "La Alianza del Pacífico en el regionalismo sudamericano actual", Revista Mexicana de Política Exterior, núm. 106 (enero-abril de 2016), p. 196, https://goo.gl/3dGVeP Es la segunda agrupación económica y comercial de América Latina y el Caribe, pero se trata de un proyecto de internacionalización, más que de una profundización del proceso de cooperación.

${ }^{18}$ La acusación añadía que la aeronave venía al "full de cocaína", Chabat, art. cit. 
tivos ideológicos (excepto Cuba), sino principalmente por razones comerciales y estratégicas.

El caso de Europa sería similar. México debía mantener un intenso diálogo político y económico con la región. El presidente viajó a Rusia, Turquía, Portugal, España y la Santa Sede. ${ }^{19}$ Mandatarios de Europa visitaron México: Suecia, Irlanda, Italia y Francia. ${ }^{20}$ Sin embargo, el mayor vínculo de México con Europa siguió siendo el Tratado de Libre Comercio con la Unión Europea (TLCUEM) de 2000. México ocupaba en ese periodo el lugar 17 como exportador para la uE y el 24 como importador. Los países de la Unión Europea en su conjunto se ubicaron como el segundo inversionista y tercer socio comercial de México, con un comercio total que ascendía a 64.3 mdd. ${ }^{21}$

En Medio Oriente, la administración quiso ampliar las relaciones con la región. Una de las acciones fue la ayuda económica destinada a los refugiados sirios por medio de la Cruz Roja y el Fondo de las Naciones Unidas para la Infancia (UNICEF), la cual le dio un lugar en la Conferencia sobre Siria en Ginebra en enero de 2014. El canciller Meade viajó a los Emiratos Árabes Unidos (EAU), Kuwait, Arabia Saudita, Jordania y Catar, donde se abrió una embajada en junio de

${ }^{19}$ En Rusia participó en la Cumbre de Líderes del Grupo de los Veinte (G20), septiembre de 2013. En Turquía, en diciembre, empezaron negociaciones para un tratado de libre comercio, la apertura de un consulado de carrera en Estambul y de una oficina de ProMéxico. José Antonio Meade, "2º Informe de Labores 2013-2014", México, SRE, 2014, p. 7, https://goo.gl/r6eSjt

${ }^{20} \mathrm{Ibid}$, p. 61. La sociedad criticó la visita del presidente François Hollande porque normalizaba las relaciones tras el caso de Florence Cassez, ciudadana francesa acusada en casos de secuestro durante el sexenio de Calderón y liberada por el gobierno de Peña. Fuentes que tuvieron acceso al expediente advirtieron sobre su inocencia y apuntaron a un arreglo de cuentas entre el hermano de ésta (Sébastien Cassez) y Genaro García Luna, entonces titular de la Secretaría de Seguridad Pública, ssP.

${ }^{21}$ Presidencia de la República, "Relación bilateral México-Unión Europea”, México, 10 de junio de 2015. 
2014. A su vez, el presidente Peña recibió en México a los mandatarios de Israel, Jordania y de los EAU. Por su parte, la agenda del gobierno mexicano para África incluyó la inauguración de dos consulados honorarios en Angola y en $\mathrm{Bu}-$ rundi, y la apertura de la Embajada de México en Doha, con nivel de Encargado de Negocios, al igual que la apertura de la Representación Diplomática en Accra, Ghana, de conformidad con los acuerdos de la Alianza del Pacífico. ${ }^{22}$

Para la región Asia-Pacífico, las prioridades económicas fueron las que guiaron la relación. Quedó claro que el acercamiento a China era primordial. Peña Nieto visitó este país en abril de 2013. En junio recibió al presidente Xi Jinping y volvieron a reunirse tres meses después durante la Cumbre de Líderes del G20, en San Petersburgo, Rusia. Se buscaba atraer inversión a México y mayor acceso a productos mexicanos en el mercado chino. En esa ocasión, China comprometió inversiones en infraestructura para comunicaciones y transportes por hasta 81000 mdd entre 2013 y 2018. Derivado de estos acuerdos, la China Railway Construction Corporation (CRCC) ganó la licitación para construir el Tren de Alta Velocidad (TAV) entre México y Querétaro, que más adelante se cancelaría.

Finalmente, una de las iniciativas más importantes para el presidente Peña Nieto en la región fue la continuidad en las negociaciones del Acuerdo Transpacífico de Cooperación Económica (o TPP por sus siglas en inglés), al que se integró en octubre de 2012. El tratado significaba una mayor sintonía y una actualización del TLCAN. Este acuerdo ayudaría a impulsar la competitividad de la planta productiva del país, además de fomentar la integración de productos mexicanos a las exportaciones de EeuU a Asia y de México a EeuU con insumos asiáticos. ${ }^{23}$

22 Meade, art. cit., pp. 87-89 y 92.

${ }^{23}$ La región del TPP representaría un mercado de más de 800 millones de consumidores (40\% del PIB mundial). Juan Salvador Navarro, "The Trans-Pacific Partnership: Understanding the Economic Impact for Mexico and Canada”, México y la cuenca del Pacífico, vol. 5, núm. 13 (2016), 
En este contexto de inicio del sexenio, en el ámbito multilateral destaca el anuncio del presidente Peña sobre el regreso de México a participar en las Operaciones de Mantenimiento de la Paz (OMP) de la ONU. ${ }^{24}$ El gobierno logró el consenso interno para esta participación que en otros tiempos enfrentó gran oposición, sobre todo de las Fuerzas Armadas.

Como puede observarse, en esta primera etapa, la política exterior de Peña Nieto fue muy activa. Hubo varias iniciativas, giras y el país estuvo presente en diversos foros internacionales. El resultado favorable en la elección de 2012 y el consenso interno que generaron las reformas estructurales permitieron una política exterior de mayor proyección. Sin embargo, esta situación cambiaría cuando ocurrieron incidentes graves en el plano interno.

Segundo periodo: de Ayotzinapa a las precampañas en EEUU

En otoño de 2014 reinó la desconfianza por parte de la población y se modificó sin remedio la percepción del gobierno mexicano en el interior y en el exterior. Un primer hecho fue el caso de los 43 normalistas desaparecidos en Iguala el 26 de septiembre de 2014, presuntamente a manos del crimen organizado coludido con policías y presidentes municipales de las ciudades de Iguala y Cocula. Este caso evidenció el total fracaso de la estrategia del gobierno para combatir la corrupción y el crimen organizado. ${ }^{25}$

pp. 27-55; Ulises Granados Quiroz, "México y el Acuerdo de Asociación Transpacífico (TPP): oportunidades y retos”, México y la cuenca del Pacífico, vol. 3, núm. 6 (2014), pp. 55-76.

${ }^{24}$ Específicamente en la $69^{\circ}$ Asamblea General de Naciones Unidas (oNU), en 2014. En abril de 2015 se inició la primera fase de participación con dos oficiales mexicanos en la Misión en Haití (minustah) y dos más a la Misión para el referéndum del Sahara Occidental (MINURso). Meade, op. cit., p. 115.

${ }^{25}$ Gracias al control de buena parte de los medios de comunicación, el gobierno de Enrique Peña desvió la atención de la seguridad a los te- 
En cuanto a la corrupción, el caso que marcó la administración fue el de la "Casa Blanca". Una fuente periodística reveló que la primera dama, Angélica Rivera, había comprado una casa de 7 mdd en la Ciudad de México. La compra se canceló con una disculpa del presidente. ${ }^{26}$ Anteriormente, una investigación realizada por la organización Mexicanos Contra la Corrupción y la Impunidad y el medio de comunicación Animal Político reveló que entre 2013 y 2014 al menos once dependencias habrían desviado dinero público a través de 186 empresas a las que el gobierno otorgó contratos irregulares, con un fraude de alrededor de 192 mdd. ${ }^{27}$ Se suman a esto los casos de los gobernadores que hicieron desfalcos millonarios a sus estados. ${ }^{28}$ Estos hechos no detuvieron las reformas estructurales, pero afectaron la imagen de México y del presidente.

mas económicos, sin reformar las instituciones de procuración de justicia ni atacar la corrupción. John M. Ackerman, "México: la transición pendiente. Déficit democrático y movilizaciones sociales", Nueva Sociedad, núm. 256 (marzo-abril de 2015), pp. 12-14, https://goo.gl/UcgqTT Anterior a Ayotzinapa estuvo el caso Tlatlaya, en el que ocho militares habrían ejecutado a quince personas en el poblado Cuadrilla Nueva, en el municipio de Tlatlaya. Mayra Zepeda, “¿Qué ocurrió en Tlatlaya minuto a minuto, según la cNDH?”, Animal Político, 22 de octubre de 2014, https:/ / goo.gl/sakTSB

${ }^{26}$ La casa fue comprada en noviembre de 2014 al empresario Juan Armando Hinojosa Cantú, dueño de grupo Higa, un contratista del gobierno cercano a Peña Nieto. Juliana Fregoso, "Los 5 escándalos de corrupción que distanciaron a Enrique Peña Nieto de los mexicanos", Infobae, 6 de septiembre de 2017, https:/ /goo.gl/MNypYV. La Encuesta Nacional de Calidad e Impacto Gubernamental 2015 reveló que 52.4\% de los ciudadanos percibía que la corrupción era muy frecuente en el gobierno, en comparación con dos años antes, cuando $42.9 \%$ expresó esto mismo.

${ }^{27}$ Mexicanos Contra la Corrupción y la Impunidad, "La estafa maestra” (en línea), México, MCCI, 2017 https://goo.gl/yE15e7

28 De 2010 a 2016, al menos 11 gobernadores fueron señalados por actos de corrupción e investigados por la Procuraduría General de la República por desvío de recursos públicos y enriquecimiento ilícito. Manuel Hernández, "El dream team de los gobernadores corruptos en México", Huffpost (edición mexicana), 29 de noviembre de 2016. 
El caso de los estudiantes desaparecidos afectó significativamente la política exterior de Peña Nieto. El primer impacto tuvo lugar días después, en la gira del presidente programada para China y Australia, en noviembre de 2014. Originalmente la extensión de la visita estaba prevista en diez días, pero "el mandatario la acortó por el caso de los 43 normalistas desaparecidos". ${ }^{29}$ El presidente no quería que, al solicitar permiso al Congreso, pudieran negarlo por el caso Ayotzinapa. ${ }^{30}$ La situación interna estaba limitando el alcance de la política exterior.

El 6 de enero de 2015, los presidentes Enrique Peña Nieto y Barack Obama se reunieron en Washington, D.C., derivado de una iniciativa ejecutiva de Eevu en materia migratoria que beneficiaría a un importante número de mexicanos. ${ }^{31}$ Los presidentes también acordaron avanzar en la instrumentación del Plan de la Alianza para la Prosperidad del Triángulo Norte con el Salvador, Guatemala y Honduras, mediante inversiones en infraestructura. La cooperación en materia fronteriza y de seguridad siguió siendo álgida. ${ }^{32}$

Con la región de América Latina y el Caribe, si bien el gobierno de Peña Nieto quiso continuar el diálogo político y reforzar el comercio, la región leyó como desinterés la cance-

29 "Peña Nieto inicia mañana viaje a Australia y China", Excélsior, 8 de noviembre de 2014. https://www.excelsior.com.mx/nacional/2014/11/ 08/991327

${ }^{30}$ De acuerdo con el artículo 88 de la Constitución mexicana, el presidente tiene que solicitar permiso al Congreso para salir del territorio más de siete días.

${ }^{31}$ Deferred Action for Childhood Arrivals (DACA) y Deferred Action for Parents of Americans and Lawful Permanent Residents (DAPA), evitarían la deportación de 5 millones de indocumentados. Daniel Villafuerte Solís, op. cit., p. 31. The New York Times destacó el diálogo que sostuvieron sobre Ayotzinapa y dio cobertura a protestas de organizaciones civiles que se oponían a la visita de Peña. Ferrer, Angélica, "Los viajes de Peña Nieto y lo que ocurría en México", Milenio, 1 de septiembre de 2015, https:// goo.gl/svFK5W

32 Ver montos de la Iniciativa Mérida en José Antonio Meade, " $3^{\circ}$ Informe de Labores 2014-2015”, México, SRE, 2015, pp. 275, 
lación de la participación del presidente en la cumbre de la Celac, mientras que acudió a foros en China y Australia en noviembre de 2014. De cualquier forma, entre septiembre de 2014 y septiembre de 2015, viajó en cinco ocasiones a países latinoamericanos y caribeños. ${ }^{33}$ Por otro lado, la inversión extranjera directa de México en la región de la Alianza del Pacífico fue de 83 mdd, más que con cualquier país del G20, con excepción de China y Estados Unidos. ${ }^{34}$

Con la Unión Europea (UE), la situación fue realmente álgida en 2014, tras los hechos de Ayotzinapa. Aunque estuvo la posibilidad, la uE no invocó la cláusula democrática, quedando claro que privilegiaba la relación comercial por encima de consideraciones sociales. ${ }^{35}$ Pese a ello, la agenda del presidente mexicano con la región fue bastante nutrida, con visitas al Reino Unido, Italia y Francia. ${ }^{36}$ La relevancia con la región en este periodo reside en que el gobierno de México y la UE acordaron, en junio de 2015, el inicio de las negociaciones de modernización del Acuerdo Global y el reforzamiento de la Asociación Estratégica.

En Asia-Pacífico, China siguió siendo prioridad. El ejecutivo realizó la tercera visita al gigante asiático en noviembre de 2014, pero la relación se tornó amarga cuando ese mismo mes el gobierno mexicano canceló la licitación para construir el Tren de Alta Velocidad México-Querétaro que había

${ }^{33}$ Aun enfrentando críticas internas por haber viajado en un tiempo muy cercano a Ayotzinapa. Rosa Elvira Vargas, "Cancela Peña participación en la cumbre de la Celac", La Jornada, 24 de enero de 2015, p. 13, https://goo.gl/nZiYbH

${ }^{34}$ Meade, "3o Informe", op. cit., pp. 275.

35 Eurodiputados de la izquierda y los verdes exigieron la invocación de la cláusula democrática, pero el Partido Popular Europeo y otros lograron detener la iniciativa. Marco Appel, "La inútil cláusula democrática del Acuerdo Global México-ue”, Proceso, 19 de abril de 2017, https:/ /goo. $\mathrm{gl} / \mathrm{nnjquy}$

36 En el Reino Unido la prensa criticó la visita del presidente y en Francia, que coincidió con el escape de prisión de Joaquín El Chapo Guzmán, France Press publicó que la visita había sido "ensombrecida" por la fuga del capo de la droga y por el caso Ayotzinapa. Angélica Ferrer, art. cit. 
ganado la China Railway Construction Corporation, ${ }^{37}$ lo que generó protestas de China y una solicitud de indemnización de 600 mdd.

\section{Tercer periodo: la irrupción de Donald Trump}

Al desgaste del gobierno del presidente Peña se sumó un acontecimiento externo. En junio de 2015, Donald Trump se registró como candidato republicano a la elección presidencial de EEUU de 2016, con discurso agresivo, entre otros, hacia México. ${ }^{38}$ Durante este periodo, la mayor cantidad de recursos y esfuerzo de México se dirigió hacia EeuU En paralelo, el cambio de gobierno en Canadá le abría la puerta al país, y Peña Nieto viajó en junio para lanzar el Diálogo Estratégico de Alto Nivel y participar en la Cumbre de Líderes de América del Norte. ${ }^{39}$

En agosto, el presidente nombró secretario de Desarrollo Social al hasta entonces canciller José Antonio Meade, en vista de las elecciones presidenciales de 2018. Claudia Ruiz Massieu Salinas llegó a ocupar la cabeza de la cancillería, quien, sin experiencia diplomática ni el oído del presidente,

${ }^{37} \mathrm{El}$ argumento fue el costo de la obra. Algunos medios publicaron que en la designación del contrato resultaron favorecidos círculos cercanos al presidente Peña. "Videgaray asesoró a empresa china en licitación del tren México-Querétaro, reporta Aristegui Noticias”, Animal Político, 7 de febrero de 2018, https:/ / goo.gl/hjRmjU . El proyecto Dragon Mart, en Cancún, Quintana Roo, de un complejo con 722 viviendas, 20 naves comerciales y 3000 locales, también se canceló bajo argumento de daños medioambientales. Rodrigo Vera, "La Profepa cancela en definitiva el proyecto Dragon Mart", Proceso, 26 de enero de 2015, https://goo.gl/aCAidj

38 Trump declaró que México enviaba a Estados Unidos "drogas, crímenes, violadores...". Carolina Martín Adalide, "El multimillonario Donald Trump se apunta a la batalla republicana", El Mundo, 16 de junio de 2015, https://goo.gl/EVmYnA

${ }^{39}$ El gobierno liberal de Justin Trudeau había decidido eliminar el requisito de visa a mexicanos. 
tuvo un desempeño positivo. ${ }^{40}$ Instrumentó una estrategia para renovar la presencia de México y el aporte de su diáspora en EeuU. ${ }^{41}$ Le tocó, además, uno de los momentos más tensos de la relación bilateral: la invitación del presidente Peña Nieto a Trump para visitar México. La visita fue muy criticada en México y fue motivo de la salida del gabinete del entonces secretario de Hacienda, Luis Videgaray.

Este contexto internacional adverso se extendió hasta las elecciones presidenciales de EEUU, en noviembre de 2016. El candidato republicano Donald Trump resultó ganador y su triunfo obligó a más cambios en la dirección de la SRE. Luis Videgaray, aún hombre fuerte del presidente, mantenía una buena relación personal con altos oficiales de aquel país, por lo que el presidente lo nombró a la cabeza de la cancillería en enero de 2017 y orientó los objetivos de política exterior hacia dos prioridades: la diversificación y un nuevo diálogo y negociación en la relación bilateral con EEUU, sobre todo en un contexto que amenazaba el TLCAN. ${ }^{42}$

Esto no bastó para evitar otro incidente. Trump firmó una acción ejecutiva que dirigía fondos hacia la construcción de un muro en la frontera con México y para reforzar las deportaciones. Como resultado, el presidente Peña can-

${ }^{40}$ Ruiz Massieu era, después de Meade, la funcionaria con más presencia en el extranjero, además de haber reposicionado a México en el top 10 de los países más visitados como secretaria de Turismo. Natalia Gómez, "Cambio en la sRe divide las opiniones", El Universal, 28 de agosto de 2015, https://goo.gl/j7Jk2W . Fue criticada, entre otros, por la declaración del presidente Peña y la canciller alemana Angela Merkel en la que se anunciaba que Alemania estaba colaborando con México para esclarecer el caso de Ayotzinapa.

${ }^{41}$ La canciller realizó 27 visitas a EEuU de marzo a octubre, para reunirse con autoridades y líderes a nivel federal, estatal y local, coordinar acciones con la Embajada de México y los 50 consulados en ese país y también recibió visitas de alcaldes, congresistas y funcionarios de EeuU. Véase Claudia Ruiz Massieu, "4º Informe de Labores 2015-2016”, México, SRE, 2016.

42 Presidencia de la República, "Objetivos de la Política Exterior de México", México, 23 de enero de 2017. 
celó el viaje planeado a EEuU el 31 de enero. ${ }^{43}$ Además, el presidente Trump había decidido que el TLCAN era desventajoso para su país por lo que, bajo amenazas de denuncia, los tres países comenzaron su renegociación a mediados de agosto de 2017 a petición del gobierno estadounidense. ${ }^{44}$

En lo referente al tema migratorio, la administración Trump continuó con el legado de su predecesor Barack Obama, por lo que México reforzó sus redes consulares y programas para recibir a los connacionales repatriados. ${ }^{45} \mathrm{En}$ la frontera sur de México, los flujos migratorios de Centroamérica seguían significando un reto para el gobierno. En este contexto, el presidente Peña Nieto moderó sus visitas a Latinoamérica y priorizó la relación comercial, con excepción de Venezuela. Siguió el impulso a la Alianza del Pacífico, aunque sus intercambios sólo representaban $1.6 \%$ de las exportaciones de México y $0.7 \%$ de las importacio-

${ }^{43}$ La reunión entre los mandatarios fue en la cumbre del G20 en julio, en Alemania. A finales de noviembre de 2018, la retórica sobre el muro volvió a tomar fuerza por la oleada de migrantes centroamericanos. El presidente Trump autorizó el despliegue de tropas militares en servicio activo a lo largo de la frontera, autorizando el uso de fuerza letal para la protección de los agentes de Aduanas y Protección Fronteriza. Bart Jansen, John Fritze y Alan Gomez, "White House approves military to use lethal force at southern border", USA Today, 21 de noviembre de 2018, https://goo.gl/4S1koG

${ }^{44}$ Las ocho rondas de negociación estuvieron bajo presión de los calendarios electorales en 2018, tanto de México, como de EeuU. En este contexto, EEUU impuso aranceles a las importaciones de acero y aluminio de México y Canadá. En paralelo, Videgaray realizó una intensa labor política. Visitas de altos oficiales de uno y otro país se relatan en el $5^{\circ}$ informe de labores de la SRE, al igual que las conversaciones telefónicas entre los presidentes. Luis Videgaray, " 5 ${ }^{\circ}$ Informe de Labores 2016-2017", México, SRE, 2017, pp. 42-48.

${ }^{45}$ Los arrestos en 2017 ascendieron a la mitad que en los años pico (2010-2011) del presidente Obama. Randy Capps, et al., "Reviving Up the Deportation Machinery: Enforcement under Trump and the Pushback", Migration Policy Institute, Washington, mayo de 2018, https://goo.gl/qeq7ZM 
nes. ${ }^{46}$ Con respecto a Venezuela, México decidió liderar una propuesta de resolución en el seno de la Organización de los Estados Americanos (OEA) ${ }^{47}$ contra el régimen de Maduro. Sin embargo, la ausencia de EeuU y el apoyo de países caribeños al gobierno represor no permitieron su aprobación.

En lo que respecta a Europa, a pesar de los incidentes de 2014 y del contexto adverso con EEUU, las visitas del presidente Peña al continente no cesaron. Asistió a diversos foros multilaterales e hizo visitas a Alemania, Dinamarca, Francia, Países Bajos y España. También recibió en México a los mandatarios de Finlandia, Dinamarca, Polonia, Alemania y Portugal, cuyas visitas tuvieron, en su mayoría, un componente comercial. Además, México y la ue, después de nueve arduas rondas de negociaciones, concluyeron exitosamente la modernización del Acuerdo Global en abril de $2018 .^{48}$

La agenda del presidente Peña para Medio Oriente fue intensa durante 2016. Para promover el comercio y las inversiones, visitó Arabia Saudita, los EAU, Kuwait y Qatar. África, por su parte, la agenda no fue muy dinámica. En lo que respecta a Asia-Pacífico, dado que Donald Trump retiró a EEUU del TPP, México, junto con los otros países que integraban el

${ }^{46}$ Las exportaciones de México hacia América Latina y el Caribe fueron de $\$ 18,240.1$ mdd en 2016, lejos de los $\$ 302,575.7$ mdd en exportaciones hacia EeuU. Secretaría de Economía (SE), "Comercio Exterior, Información Estadística y Arancelaria, Importaciones y exportaciones por sector país 2009-2016, por acuerdos", México, SE, 2015, https://goo. $\mathrm{gl} / \mathrm{Lj} 6 \mathrm{vyS}$

47 Para junio de 2017, Venezuela tenía una tasa de inflación de más de $400 \%$, una deuda de $\$ 7,200$ mdd y durante ese año la economía registró un decrecimiento de $8 \%$, además de un saldo de 61 muertos por las protestas contra el gobierno de Maduro. La resolución presentada solicitaba al régimen de Maduro respetar los derechos humanos, abandonar la convocatoria de la Asamblea Constituyente y establecer un calendario electoral. "Venezuela vive la peor crisis económica de su historia: Al Yazira”, Excélsior, 2 de junio de 2017, https://goo.gl/ZRrFwV

48 "México-Unión Europea", Sistema de Información sobre el Comercio Exterior (sitio de internet), Organización de los Estados Americanos (OEA), 2018, https://goo.gl/W8rT3k 
acuerdo, comenzaron conversaciones para concretar el instrumento comercial que se firmó en marzo de $2018 .{ }^{49}$

Con respecto a China, y aun con los desencuentros derivados de la cancelación del contrato del tren rápido y el desarrollo en Quintana Roo, en junio de 2016 el Banco de Industria y Comercio de China (ICBC) y el Banco de China iniciaron operaciones en México. En 2017, México tuvo un déficit récord con China de 67432 mdd. $^{50}$

Finalmente, en el ámbito multilateral destaca la participación de México en las negociaciones de la Agenda de Desarrollo Post-2015, adoptada en septiembre de 2015; el aumento de la participación de efectivos mexicanos en las OMP; la firma del Acuerdo de París sobre Cambio Climático que México ratificó en septiembre de 2016 , y la conmemoración del $50^{\circ}$ Aniversario de la firma del Tratado de Tlatelolco, en febrero de 2017, en la Ciudad de México. ${ }^{51}$

Cuarto periodo: las elecciones del primero de julio de 2018 y el no-gobierno de EPN

El $1^{\circ}$ de julio de 2018, el candidato de Morena, Andrés Manuel López Obrador, ganó las elecciones presidenciales con $53.19 \%$ de los votos, según datos del Instituto Nacional Elec-

49 "Comprehensive and Progressive Agreement for Trans-Pacific Partnership (TPP-11)", Australian Government, Department of Foreign Affairs and Trade; los integrantes del TPP 11 son: Australia, Brunei Darussalam, Canadá, Chile, Japón, Malasia, México, Perú, Nueva Zelanda, Singapur y Vietnam.

${ }^{50}$ China es el segundo socio comercial de México, segunda fuente de importaciones y tercer destino de exportaciones. Abraham Zamora, "Oportunidades de inversión en México: infraestructura y zEE", en Enrique Dussel (coord.), La relación México-China. Desempeño y propuestas para 2016-2018, México, UnAm, 2016, pp. 49-55, https://goo.gl/8GAaZP ; "8 puntos que debes conocer de la relación México-China", Expansión, 3 de agosto de 2018, https://goo.gl/dpZewA

${ }^{51}$ Claudia Ruiz Massieu, op. cit., pp. 322; Luis Videgaray, op. cit., pp. 316. 
toral (INE). Esto marcó el último periodo de Enrique Peña Nieto. El tema más relevante del periodo en la relación con América del Norte fue la conclusión del nuevo tratado comercial (T-MEC). El 27 de agosto de 2018 se concluyó la negociación entre México y EEUU, pues había una fuerte presión por formalizar un acuerdo antes del cambio de gobierno. ${ }^{52}$ Canadá se unió a lo ya pactado en octubre. El 30 de noviembre de 2018 se firmó el tratado en Buenos Aires, Argentina. ${ }^{53}$ El presidente electo, que en el pasado había sido acérrimo crítico de las políticas de libre mercado, elogió las negociaciones de última hora que salvaron el acuerdo trilateral.

Finalmente, la relación con China también se ha considerado importante para López Obrador. El 5 de noviembre envió a su equipo económico a Shanghái, respondiendo a la invitación del gobierno chino a una feria comercial.

\section{Conclusiones}

En general, el presidente Enrique Peña realizó 68 viajes durante su mandato que resultaron en 94 encuentros. Dos terceras partes los hizo después de los hechos de Ayotzinapa y con críticas dentro y fuera del país. Aun cuando no hubo acciones extraordinarias de política exterior, la instrumentación ordinaria de ésta no se detuvo. También hubo continuidad en la política exterior como política económica

52 Videgaray habría comentado que el acuerdo entre México y EEuU se materializaría, "independientemente de lo que ocurr[iera] con Canadá”. Ángel Bermúdez, "Negociación del TLCAN: por qué Estados Unidos y México dejaron de lado a Canadá”, BBC Mundo, 29 de agosto de 2018, https:/ /goo.gl/SA4YtR Cabe señalar que en el equipo negociador mexicano participó Jesús Seade en representación del presidente electo López Obrador.

53 También se dio la Orden Mexicana del Águila Azteca a Jared Kushner, yerno y asesor del presidente Donald Trump, por su trabajo en la negociación del T-MEC, desatando polémica en redes sociales y medios de comunicación. 
con la renegociación del TLCAN, el impulso a la Alianza del Pacífico, la renegociación del TLCUEM y la firma del TPP-11.

El objetivo de diversificación no se logró plenamente, debido a que la prioridad fue fortalecer el acuerdo trilateral con Estados Unidos y Canadá. ${ }^{54}$ México ingresó al TPP, pero el volumen comercial no cambió desde una perspectiva geográfica. Asimismo, el país tuvo una fuerte presencia en América Latina mediante la Alianza del Pacífico. Sin embargo, su presencia en este mecanismo de integración regional no modificó los números de comercio e inversiones de manera significativa.

Respecto a los pilares de política exterior que planteó el programa sectorial de la SRE, el sexenio de Peña Nieto inició con una fuerte presencia del país en el mundo. Parecía que el objetivo de convertirse en un actor globalmente responsable podría cumplirse. Sin embargo, los problemas internos a mitad del sexenio y la aparición de Donald Trump impidieron que México pudiera alcanzar ese objetivo. En relación con la cooperación internacional, el gobierno peñista trató de impulsar una mayor colaboración del país. Sin embargo, la falta de un amplio presupuesto impidió alcanzar esa meta de manera cabal. El país cooperó con algunos países, especialmente de Centroamérica, pero los alcances de la contribución fueron limitados.

En cuanto a los objetivos de política exterior planteados en el Plan Nacional de Desarrollo, hubo altibajos. México impulsó en diversos foros multilaterales un diálogo político para ampliar y fortalecer su presencia en el mundo. Sin embargo, al final del sexenio la evaluación no es altamente positiva debido a que la imagen negativa del país por la corrupción y la violencia afectó su imagen en el exterior. Asimismo, la administración peñista promovió el valor de México en el mundo mediante la difusión económica, turística y cultural

${ }^{54}$ Francisco de Rosenzweig, "La tan añorada diversificación”, El Universal, 22 de octubre de 2017, https://goo.gl/Cy7vPd 
del país. Sin embargo, la actividad no se reflejó en una mayor atracción de inversiones y divisas.

En cuanto al tercer objetivo del PND, el gobierno de Peña Nieto logró reafirmar el compromiso del país con el libre comercio, la movilidad de capitales y la integración productiva. Durante el sexenio, tanto la SRE como la Secretaría de Economía hicieron esfuerzos destacados para mantener una política de puertas abiertas al libre comercio y la atracción de inversiones.

Asimismo, la SRE realizó esfuerzos significativos para velar por los intereses de los mexicanos en el extranjero. La principal labor de las embajadas y consulados mexicanos estuvo relacionada con esa meta. Sin embargo, las capacidades de la cancillería se ven limitadas debido al gran número de mexicanos que demandan protección y servicios, y a la falta de mayores recursos humanos y económicos.

Este texto ha dejado evidencia de que la política exterior de Enrique Peña Nieto fue relativamente activa al principio del sexenio. El resultado de la elección presidencial le permitió al nuevo presidente un margen más amplio de acción, puesto que las relaciones exteriores de México no estarían atadas a la situación interna. Gracias a esta situación, el país empezó a mejorar gradualmente su imagen en el exterior. Las reformas constitucionales en las áreas fiscal, energética, educativa y otras trajeron una mayor atención del mundo en México. Sin embargo, antes de la mitad del sexenio la situación se modificó significativamente. Los casos de violaciones a los derechos humanos, la constante violencia en el país, la corrupción rampante en varios niveles de gobierno, la baja del precio del petróleo y la devaluación del peso frente al dólar impactaron de manera negativa la imagen de México en el exterior. Ello afectó la política exterior de México y el país recibió críticas fuertes de la comunidad internacional.

En la parte final del sexenio de Peña Nieto, la política exterior regresó a la lista de prioridades debido a la llegada del presidente Donald Trump a la presidencia de EEUU. La amenaza que representó la posible cancelación del TLCAN y 
la construcción del muro en la frontera obligó al gobierno peñista a replantear sus estrategias frente al vecino del norte.

La principal conclusión entonces es que la política exterior de México se convierte en una prioridad como respuesta a estímulos externos. Por lo tanto, las relaciones exteriores de México en algunos casos son reactivas frente a coyunturas específicas y no existe un ejercicio de planeación continuo. La política exterior de México en el sexenio de Peña Nieto empezó buscando mejorar la imagen del país en el mundo, pero pronto fue claro que el factor externo y las cuestiones internas no permitieron la priorización de la política exterior.

\section{REFERENCIAS BIBLIOGRÁFICAS}

"8 puntos que debes conocer de la relación México-China”, Expansión, 3 de agosto de 2018, https://goo.gl/dpZewA

Ackerman, John M., "México: la transición pendiente. Déficit democrático y movilizaciones sociales”, Nueva Sociedad, núm. 256 (marzo-abril de 2015), pp. 4-16, https://goo.gl/UcgqTT

Agencia Mexicana de Cooperación Internacional para el Desarrollo (Amexcid), Cooperación de México con Centroamérica y el Caribe, México, Amexcid, 12 de abril de 2018, https:/ /goo. $\mathrm{gl} / \mathrm{xEkQ96}$

Alcocer, Sergio, "Las relaciones de México con América del Norte", en 1 er Informe de Labores 2012-2013, México, sRE, https:/ / www.gob.mx/cms/uploads/attachment/file/103256/Primer_ Informe_de_Labores_SRE_2013.pdf (consulta del 12 de mayo de 2018).

Appel, Marco, "La inútil cláusula democrática del Acuerdo Global México-UE", Proceso, 19 de abril de 2017, https:/ / goo.gl/nnjquy Barrientos, Fernando y Daniel AÑorve, "México 2013: acuerdos, reformas y descontento". Revista de ciencia politica (Santiago), vol. 34, núm.1 (2014), pp. 221-247, doi: 10.4067/S0718-090X20 14000100011 
Bermúdez, Ángel, "Negociación del tlcan: por qué Estados Unidos y México dejaron de lado a Canadá”, BBC Mundo, 29 agosto 2018, https://goo.gl/SA4YtR

Capps, Randy, Muzaffar Chishti, Julia Gelatt, Jessica Bolter y Ariel G. Ruiz Sото, "Revving Up the Deportation Machinery: Enforcement under Trump and the Pushback", Migration Policy Institute, Washington, mayo de 2018, https://goo.gl/ qeq7ZM

Chabat, Jorge, "La política exterior de México: de Calderón a Peña Nieto”, Adrián Bonilla y Grace Jaramillo (eds.), La CELAC en el escenario contemporáneo de América Latina y del Caribe, San José, Costa Rica, Flacso, 2014, pp. 27-43.

"Comprehensive and Progressive Agreement for Trans-Pacific Partnership (TPP-11)”, Australian Government, Department of Foreign Affairs and Trade.

"Diálogo Económico de Alto Nivel", México-sre, s/f, https://goo. $\mathrm{gl} / \mathrm{BsKv} 6 \mathrm{~A}$

Ferrer, Angélica, "Los viajes de Peña Nieto y lo que ocurría en México", Milenio, 1 de septiembre de 2015, https://goo.gl/ svFK5W

Franco, Fernando, "México, el socio no europeo más importante de España”, Milenio, 12 de octubre de 2016, https://goo. $\mathrm{gl} / 5 \mathrm{MrqJY}$

Fregoso, Juliana, "Los 5 escándalos de corrupción que distanciaron a Enrique Peña Nieto de los mexicanos", Infobae, 6 de septiembre de 2017, https:/ / goo.gl/MNypYV

Gabriel, Dana, "The Next Phase of North American Integration. NAFTA to be Swallowed up by the Trans-Pacific Partnership (TPP)?”, Global Research, 2014, http://goo.gl/Fbah4f

Gómez, Natalia, "Cambio en la sRe divide las opiniones", El Universal, 28 de agosto de 2015, https://goo.gl/j7Jk2W

Granados, Ulises, "México y el Acuerdo de Asociación Transpacífico (TPP): oportunidades y retos", México y la cuenca del Pacifico, vol. 3, núm. 6 (2014), pp. 55-76.

Hernández, Manuel, "El dream team de los gobernadores corruptos en México", Huffpost edición mexicana, 29 de noviembre de 2016. 
Jansen, Bart, John Fritze y Alan Gómez, "White House approves military to use lethal force at southern border", USA Today, 21 de noviembre de 2018, https:/ / goo.gl/4S1koG

Leví Coral, Michel y Giulliana Reggiardo, "La Alianza del Pacífico en el regionalismo sudamericano actual", Revista Mexicana de Política Exterior, núm. 106 (enero-abril de 2016), pp. 187-204, https://goo.gl/3dGVeP

Limón, Mónica Vanessa, "La relación entre México y Cuba", Comercio Exterior, vol. 64, núm. 2 (marzo y abril de 2014), p. 11, https://goo.gl/8Vx5f5

Lozoya, Emilio y Jorge Montaño, "Una visión de México para el futuro. Sección especial: Propuestas para la próxima política exterior de México", Foreign Affairs Latinoamérica, vol. 12, núm. 2 (2012), pp. 43-51.

Martín Adalide, Carolina, "El multimillonario Donald Trump se apunta a la batalla republicana", El Mundo, 16 de junio de 2015, https://goo.gl/EVmYnA

Mexicanos Contra la Corrupción y la Impunidad, "La estafa maestra”, México, Mcci, 2017 (documento en línea), https://goo. $\mathrm{gl} / \mathrm{yE} 15 \mathrm{e} 7$

MeAde, José Antonio, "2o Informe de Labores 2013-2014”, México, SRE, 2014, 276 pp., https://goo.gl/r6eSjt

Meade, José Antonio, “3o Informe de Labores 2014-2015”, México, SRE, 2015, 275 pp., https://goo.gl/r6eSjt

Meléndez, José, "México-Cuba: una relación con altibajos", El Universal, 27 de enero de 2014, http:/ /goo.gl/SJvKyt

Navarro, Juan Salvador, "The Trans-Pacific Partnership: Understanding the Economic Impact for Mexico and Canada", México y la cuenca del Pacífico, 2016, vol. 5, núm. 13, pp. 27-55.

Notimex, "Venezuela vive la peor crisis económica de su historia: Al Yazira”. Excélsior, 2 de junio de 2017, https:/ / goo.gl/ZRrFwV Organización de los Estados Americanos (oEA), "México-Unión Europea”, Sistema de Información sobre el Comercio Exterior, 2018. https://goo.gl/W8rT3k

Plan Nacional de Desarrollo (PND), 2013-2018, Diario Oficial de la Federación, 20 de mayo de 2013, http://goo.gl/kxDcCS 
Presidencia de la República "Objetivos de la Política Exterior de México", 23 de enero de 2017.

Presidencia de la República, "Relación bilateral México-Unión Europea”, México, 10 de junio de 2015. https://goo.gl/9sjc7j

Ribando Seelke, Clare y Kristin Finklea, "U.S.-Mexican Security Cooperation: The Mérida Initiative and Beyond", 7-5700. R41349, US, Congressional Research Service, 7 de mayo de 2015, https://goo.gl/QufDbG

Romero, María Elena y Óscar Rivera, "Los retos actuales de la política exterior mexicana: una revisión desde la geopolítica crítica", Asian Journal of Latin American Studies, vol. 26, núm. 3 (2013), pp. 1-28.

Rosenzweig, Francisco de, "La tan añorada diversificación", $E l$ Universal, 22 de octubre de 2017, https://goo.gl/Cy7vPd

Secretaría de Economía (SE), "Comercio Exterior, Información Estadística y Arancelaria, Importaciones y exportaciones por sector país 2009-2016, Por acuerdos”, se, 25 de mayo de 2015, https://goo.gl/Lj6vyS

Secretaría de Relaciones Exteriores (SRE), "México y Canadá: una relación amplia y profunda”, México, sRe, 14 de octubre de 2015, https://goo.gl/eTTRFV

TAPIA, José, "El gasto en Seguridad. Observaciones de la ASF a la gestión y uso de recursos", México Evalúa, Centro de Análisis en Políticas Públicas, A.C., junio de 2011, https://goo.gl/z2dcsy

VArgas, Rosa Elvira, "Cancela Peña participación en la cumbre de la CELAC", La Jornada, 24 de enero de 2015, p. 13, https:/ / goo. $\mathrm{gl} / \mathrm{nZiYbH}$

Vera, Rodrigo, "La Profepa cancela en definitiva el proyecto Dragon Mart", Proceso, 26 de enero de 2015, https:/ / goo.gl/aCAidj "Videgaray asesoró a empresa china en licitación del tren MéxicoQuerétaro, reporta Aristegui Noticias", Animal Político, 7 de febrero de 2018, https://goo.gl/hjRmjU

Villafuerte, Daniel, "La política migratoria en tiempos de Obama: implicaciones en la frontera sur de México", Ciencias Sociales y Humanidades, vol. 4, núm. 1 (2017), pp. 29-44.

Zamora, Abraham, "Oportunidades de inversión en México: infraestructura y ZEE”, en Enrique Dussel (coord.), La relación 
México-China. Desempeño y propuestas para 2016-2018, México, UNAM, 2016, pp. 49-55, https://goo.gl/8GAaZP

ZePEDA, Mayra, “¿Qué ocurrió en Tlatlaya minuto a minuto, según la cNDH?”, Animal Político, 22 de octubre de 2014, https:/ / goo. $\mathrm{gl} / \mathrm{sakTSB}$

Informes, leyes e instrumentos internacionales

Gobierno de México y Gobierno de Canadá, Alianza México-Canadá, reporte anual, 2012-2013, https://goo.gl/WPMdWs

Ruiz Massieu Salinas, Claudia, $4^{\circ}$ Informe de Labores 2015-2016, Secretaría de Relaciones Exteriores, México, SRE, 2016, 322 pp. Videgaray, Luis, $5^{\circ}$ Informe de Labores 2016-2017, México, SRE, 2017, 316 pp. 
